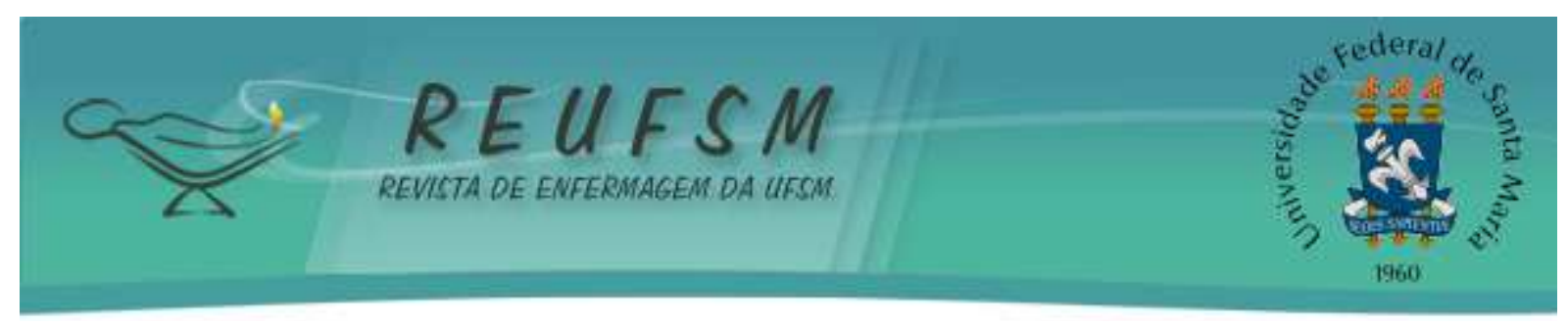

\title{
PERFIL SOCIODEMOGRÁFICO E OBSTÉTRICO DE MULHERES EM IDADE MATERNA AVANÇADA
}

\author{
SOCIODEMOGRAPHIC AND OBSTETRIC PROFILE OF WOMEN IN ADVANCED \\ MATERNAL AGE
}

PERFIL SOCIODEMOGRÁFICO Y OBSTÉTRICO DE MUJERES EN EDAD MATERNA AVANZADA

Doi: $10.5902 / 2179769225922$

\author{
Juliane Dias Aldrighi ${ }^{1}$ \\ Suelen da Silva Ribeiro ${ }^{2}$ \\ Marilene Loewen Wall ${ }^{3}$ \\ Samuel Spiegelberg Züge ${ }^{4}$ \\ Silvana Regina Rossi Kissula Souza ${ }^{5}$ \\ Adriana Aparecida Piler ${ }^{6}$
}

RESUMO: Objetivo: descrever o perfil sociodemográfico e obstétrico de mulheres em idade materna avançada atendidas em 2014 em um hospital universitário do sul do Brasil. Método: pesquisa descritiva, retrospectiva, de abordagem quantitativa, constituída de 223 prontuários de mulheres. Os dados foram coletados por meio de um questionário estruturado no período de setembro de 2015 a janeiro de 2016. Resultados: das mulheres, 68,6\% tinham entre 35 e 39 anos, $82,5 \%$ brancas, $75,7 \%$ casadas, $35,4 \%$ tinham ensino médio completo e $51,1 \%$ exerciam trabalho remunerado. Quanto ao perfil obstétrico, 91,9\% realizaram o pré-natal, $53,4 \%$ tiveram parto cesáreo, $75,3 \%$ tiveram bebê a termo. Das 75,8\% que apresentaram complicações, $14,2 \%$ tiveram pré-eclâmpsia e $28,4 \%$ diabetes mellitus gestacional. Considerações finais: os dados evidenciam um perfil sociodemográfico de mulheres brancas, com baixo nível de escolaridade e baixa renda. $\mathrm{Na}$ perspectiva obstétrica, houve alta porcentagem de complicações associadas à própria idade e às doenças pré-gestacionais.

Descritores: Enfermagem; Idade materna; Complicações na gravidez

ABSTRACT: Aim: to describe the sociodemographic and obstetric profile of women in advanced maternal age, who were cared for in a university hospital in 2014. Method: it is a descriptive research, with quantitative approach and retrospective design. Data collection was held in medical charts of 223 women through a structured questionnaire, from September 2015 to January 2016. Results: among the women, 68.6\% were aged between 35 and 39 years-old, $82.5 \%$ were white, $75.7 \%$ were married, $35.4 \%$ completed high school and $51.1 \%$ had paid jobs. Concerning the obstetric profile, $91.9 \%$ underwent prenatal care, $53.4 \%$ had

\footnotetext{
${ }^{1}$ Enfermeira. Mestre em Enfermagem. Universidade Federal do Paraná. Curitiba, PR, Brasil. E-mail: juliane.aldrighi@gmail.com

${ }^{2}$ Enfermeira. Universidade Federal do Paraná. Curitiba, PR, Brasil. E-mail: ssr.suelen@ gmail.com

${ }^{3}$ Enfermeira. Doutora em Enfermagem. Universidade Federal do Paraná. Curitiba, PR, Brasil. E-mail: wall@ufpr.br

${ }^{4}$ Enfermeiro. Doutorando em Enfermagem. Universidade Comunitária da Região de Chapecó. Chapecó, SC, Brasil. E-mail: samuelzuge@gmail.com

${ }^{5}$ Enfermeira. Doutora em Enfermagem. Universidade Federal do Paraná. Curitiba, PR, Brasil. E-mail: skissula@ufpr.br

${ }^{6}$ Enfermeira. Mestre em Enfermagem. Universidade Federal do Paraná. Curitiba, PR, Brasil. E-mail: apiler@gmail.com
} 


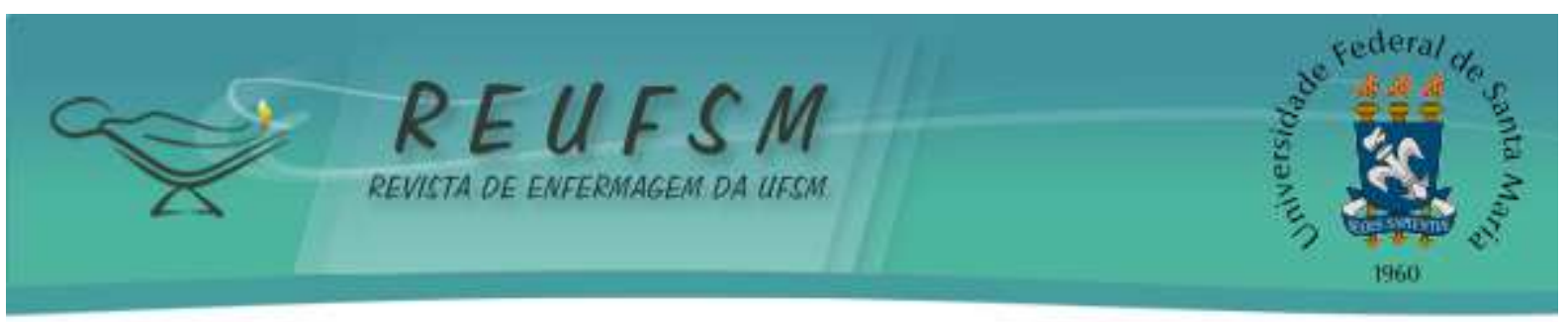

cesarean deliveries and $75.3 \%$ had full-term. As for complications, $75.8 \%$ showed some complication during pregnancy, such as pre-eclampsia (14.2\%) and gestational diabetes mellitus (28.4\%). Final considerations: the data showed a sociodemographic profile of white women, with low educational level and low income. In the obstetric perspective, there was a high percentage of complications associated with age and pre-gestational diseases.

Descriptors: Nursing; Maternal age; Pregnancy complications

RESUMEN: Objetivo: describir el perfil sociodemográfico y obstétrico de mujeres en edad materna avanzada atendidas en 2014 en un hospital universitario del sur de Brasil. Método: investigación descriptiva, retrospectiva, de perspectiva cuantitativa, constituida por 223 registros médicos de mujeres. La recolección de datos ocurrió por medio de un cuestionario estructurado, desarrollado entre septiembre 2015 y enero 2016. Resultados: de esas mujeres investigadas, 68,6\%, tenían entre 35 y 39 años, 82,5\% eran blancas, 75,7\% casadas, 35,4\% concluyeron la enseñaza secundaria y 51,1\% tenían empleo remunerado. Sobre el perfil obstétrico, 91,9\% tenían atención prenatal, 53,4\% tuvieron partos por cesárea, 75,3\% tuvieron bebés términos. De las $75,8 \%$ que presentaron complicaciones, $14,2 \%$ tuvo preeclampsia y 28,4\% de la diabetes mellitus gestacional. Consideraciones finales: los datos evidenciaron un perfil sociodemográfico de mujeres blancas, con bajo nivel de escolaridad y bajos ingresos. En la perspectiva obstétrica se observó un alto porcentaje de complicaciones asociadas a la edad y a las enfermedades pregestacionales.

Descritores: Enfermería; Edad materna; Complicaciones del embarazo

\section{INTRODUÇÃO}

A gravidez tardia é compreendida como aquela iniciada após os 35 anos, e é classificada de alto risco por ter a chance de desenvolver complicações como diabetes mellitus gestacional, pré-eclâmpsia, trabalho de parto prematuro. Nessa idade, é comum mulheres passarem por modificações fisiológicas que, eventualmente, podem estar associadas a alterações patológicas por isso, favorecem a diminuição da fertilidade, dificuldades no trabalho de parto e parto, aumentando o risco para abortos, hemorragias e o desenvolvimento de doenças genéticas e crônicas, como a síndrome hipertensiva específica da gravidez e a eclampsia. ${ }^{1}$

O Ministério da Saúde (MS) corrobora tal informação afirmando que, além de ter uma chance maior de vivenciar complicações, se não houver interferências por meio da equipe de saúde, tanto a gestante, quanto seu bebê podem evoluir para um desfecho negativo, como o óbito materno, fetal ou neonatal. ${ }^{2}$

No entanto, a gestação tardia tem sido uma escolha cada vez mais recorrente nos dias atuais. Conforme a literatura, esse fato está baseado na melhoria da qualidade de vida, no aperfeiçoamento dos métodos contraceptivos e na busca pelo parceiro certo. Atualmente, a 


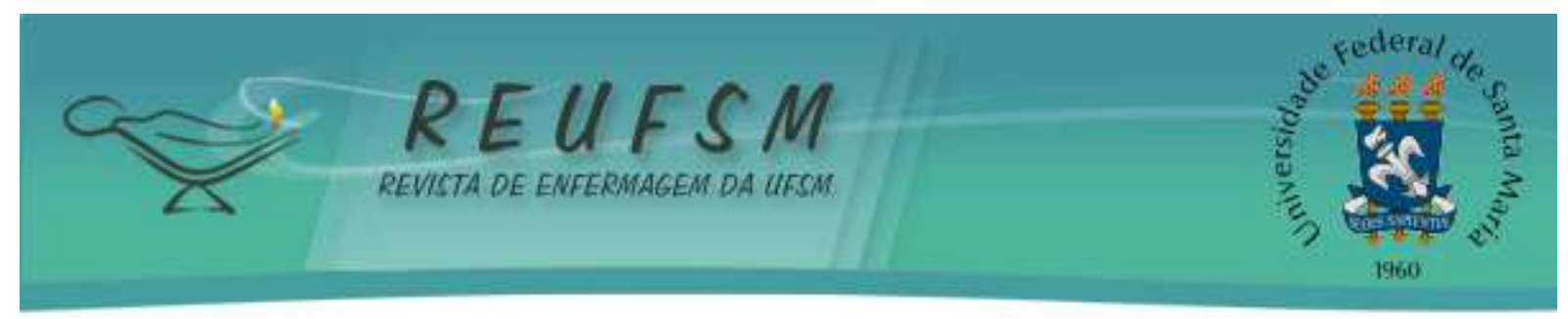

mulher dedica seu tempo à carreira e trabalho e, mesmo com a intenção de ter filhos, a gestação passa a ser deixada para o futuro. ${ }^{3-4}$

Em países desenvolvidos, há 20 anos é observado o crescente número de mulheres que têm seu primeiro filho com idade superior aos 35 anos. Um dos fatores atribuídos a esse evento é a eficácia dos métodos contraceptivos, o que auxilia a mulher na decisão do momento certo para a gestação. ${ }^{5}$ Um estudo identificou a média da idade fértil de mulheres na Inglaterra e no País de Gales, e constatou que, no ano de 1975, essa média era de 26,4 anos, já em 2013, as mulheres tinham seu primeiro filho aos 30 anos de idade. ${ }^{6}$

Uma pesquisa realizada por meio do banco de dados de Tecnologia da Informação do Serviço do Sistema Único de Saúde (DATASUS), com mães das Regiões Norte, Nordeste, Centro-Oeste, Sudeste e Sul do Brasil, avaliou a frequência de gestação em mulheres com idade superior a 34 anos entre 2006 e 2012. Verificou-se que o número de mulheres gestantes nessa idade aumentou $18,1 \%$ vezes no período e houve uma redução do número total de nascidos vivos. Apenas em 2012, as gestações em idade materna tardia representaram 11,28\% do total de partos. ${ }^{7}$ Esse dado está em conformidade com o encontrado na literatura internacional, que também declara que a proporção de parturições em mulheres em idade avançada é de, aproximadamente, $11 \% .^{5}$

Percebe-se que o Brasil segue uma tendência mundial de postergação da gestação, evidenciando uma mudança nos padrões reprodutivos, atrelada à transição demográfica e à consequente transição da fecundidade. Dessa forma, a enfermagem possui atuação importante no cuidado às gestantes, pois realiza o cuidado pré-natal que engloba planejamento, intervenções e avaliação, sendo que o perfil sociodemográfico e obstétrico das mulheres em idade tardia se torna relevante, pois, ao conhecer essas características e os desfechos apresentados pela gestação nessa idade, pode-se pensar em intervenções que contribuirão para a melhoria do cuidado em enfermagem.

Diante do exposto, o presente estudo teve como questão norteadora: quais as características sociodemográficas e obstétricas de mulheres com idade materna avançada que tiveram partos no ano de 2014 em um hospital universitário de referência no sul do Brasil? E, como objetivo: descrever o perfil sociodemográfico e obstétrico de mulheres em idade materna avançada atendidas em 2014 em um hospital universitário do sul do Brasil. 


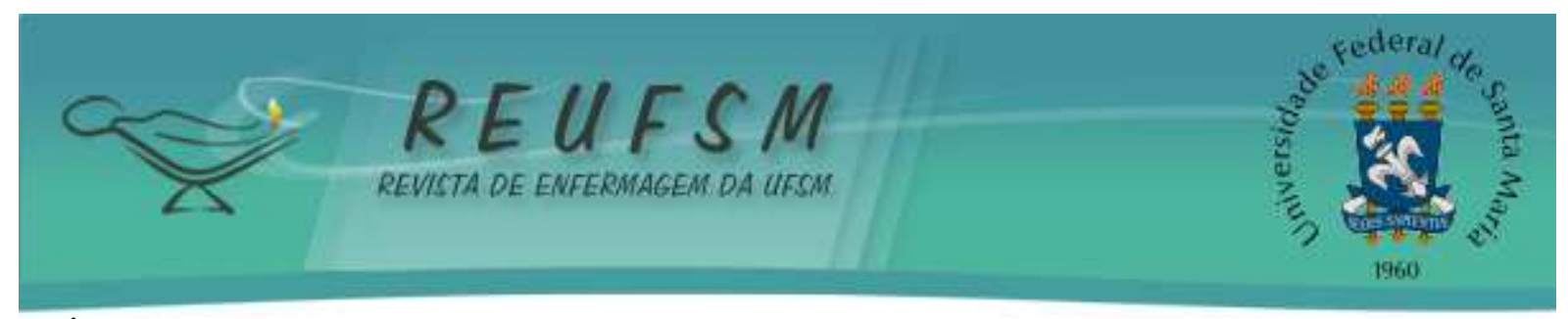

\section{MÉTODO}

Trata-se de uma pesquisa do tipo descritiva, com abordagem quantitativa, de delineamento retrospectivo. Os critérios de inclusão foram: mulheres com 35 anos ou mais, que tiveram seu parto em 2014, em um hospital universitário de referência no Sul do Brasil. Foram identificados 1442 nascimentos em 2014 no referido hospital. Desses, 223 prontuários foram selecionados, pois pertenciam a mulheres com idade de 35 anos ou mais. $\mathrm{O}$ recorte temporal foi estabelecido considerando que este estudo faz parte de um projeto iniciado em 2015, com coletas anuais de dados de mulheres que tiveram partos com idade igual ou superior a 35 anos, a fim de montar um banco de dados para a construção de uma série histórica quanto às características dessa população.

Os dados foram coletados por meio de um questionário estruturado, entre setembro de 2015 e janeiro de 2016. O instrumento de coleta de dados estava composto por 25 perguntas de múltipla escolha, que abrangeram aspectos sociodemográficos (idade; estado civil; cor/raça; escolaridade; profissão/ocupação), histórico obstétrico (idade gestacional; número de consultas durante o pré-natal; paridade; tipo de gestação; tipo de parto; complicação na gestação atual) e dados da última gestação (doenças pré-gestacionais; complicações na gestação anterior; filhos com malformação). O item complicações, tanto na gestação atual quanto nas anteriores (se houvesse), assim como os itens doenças pré-gestacionais e complicações nas gestações anteriores poderiam ter mais de uma opção marcada.

A coleta foi operacionalizada por uma aluna bolsista de Programa de Iniciação Científica do Conselho Nacional de Desenvolvimento Científico e Tecnológico (CNPq) e outra do mestrado acadêmico em Enfermagem da Universidade Federal do Paraná (UFPR). Estas foram devidamente capacitadas por meio da apresentação do projeto, o papel de cada uma na pesquisa e um vídeo explicativo quanto ao uso do software Epi Info, utilizado para a coleta de dados informatizada. As alunas assistiram ao vídeo e, em data agendada, puderam sanar dúvidas e simular a coleta dos dados com o formulário já inserido no software.

A partir disso, contatou-se o Departamento de Planejamento do hospital que disponibilizou uma lista contendo o total de partos na instituição em 2014 e os registros de todas as mulheres com idade igual ou maior que 35 anos que tiveram partos nesse período. $\mathrm{Na}$ sequência, os prontuários foram solicitados ao Departamento de Arquivo do referido hospital universitário, o qual os disponibilizou juntamente ao espaço físico próprio para coleta de dados. 


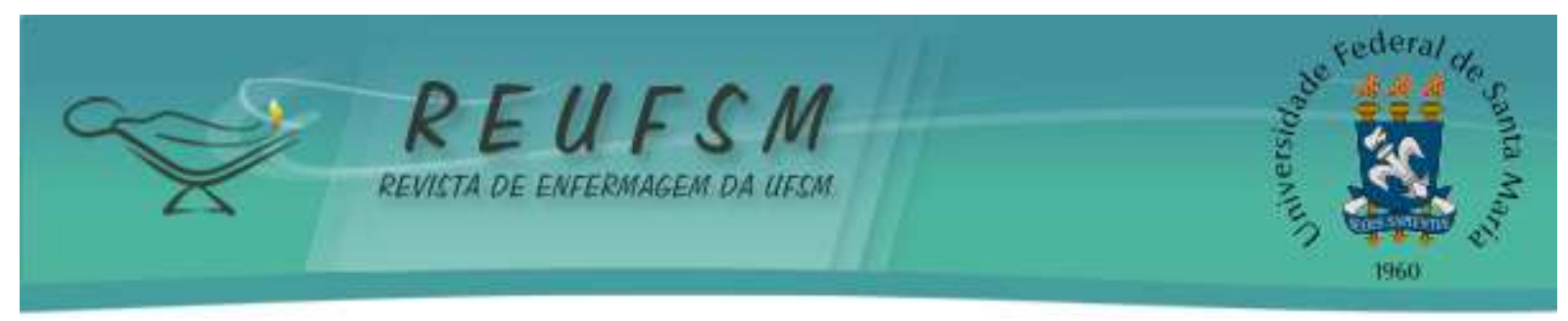

Após a coleta, os dados foram digitados e analisados por meio de estatística descritiva simples com o auxílio do programa Statistical Product and Service Solutions (SPSS), versão 18.0.

A pesquisa faz parte de um projeto maior, o qual foi aprovado pelo Comitê de Ética em Pesquisa do Hospital de Clínicas da UFPR, sob o Parecer $n^{\circ} 1.155 .166$ e registro CAAE: 46154615.7.0000.0096, de 21 de julho de 2015. Os autores conduziram a pesquisa conforme os padrões éticos exigidos pela Resolução do Conselho Nacional de Saúde $\mathrm{n}^{\circ} 466$ de 12 de dezembro de 2012.

\section{RESULTADOS}

Quanto aos dados sociodemográficos, $153(68,6 \%)$ tinham idade entre 35 e 39 anos, $169(75,8 \%)$ casadas ou em união estável, 184 (82,5\%) eram brancas, 151 (67,7\%) residiam em Curitiba, 79 (35,4\%) tinham ensino médio completo e 76 (34\%) trabalhavam no serviço de limpeza e vendedoras do comércio (Tabela 1).

Tabela 1 - Características sociodemográficas das mulheres de idade materna avançada que tiveram partos em um hospital-escola do Sul do Brasil no ano de 2014. Curitiba/PR, Brasil, 2017. ( $\mathrm{N}=223)$

\begin{tabular}{|c|c|c|}
\hline Variável & $\mathrm{N}$ & $\%$ \\
\hline \multicolumn{3}{|c|}{ Faixa etária em anos } \\
\hline 35 a 39 anos & 153 & 68,6 \\
\hline 40 a 44 anos & 62 & 27,9 \\
\hline 45 anos ou mais & 8 & 3,5 \\
\hline \multicolumn{3}{|l|}{ Estado Civil } \\
\hline Solteira & 46 & 20,6 \\
\hline Divorciada/Separada & 6 & 2,7 \\
\hline Viúva & 1 & 0,4 \\
\hline Casada & 169 & 75,8 \\
\hline Ignorado & 1 & 0,4 \\
\hline \multicolumn{3}{|c|}{ Município de Residência } \\
\hline Curitiba & 151 & 67,7 \\
\hline Outros & 72 & 32,2 \\
\hline \multicolumn{3}{|l|}{ Cor/Raça } \\
\hline Branca & 184 & 82,5 \\
\hline Preta & 25 & 11,2 \\
\hline Amarela & 1 & 0,4 \\
\hline Parda & 4 & 1,8 \\
\hline Ignorada & 8 & 3,6 \\
\hline Outra & 1 & 0,4 \\
\hline
\end{tabular}




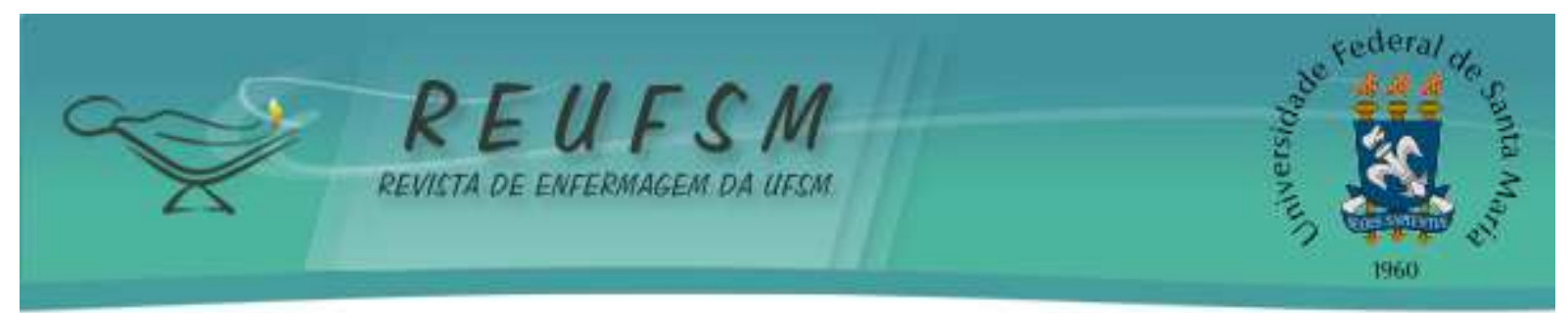

Escolaridade

\begin{tabular}{|c|c|c|}
\hline Analfabeta & 1 & 0,4 \\
\hline Ensino Fundamental Incompleto & 34 & 15,2 \\
\hline Ensino Fundamental Completo & 37 & 16,6 \\
\hline Ensino Médio Incompleto & 10 & 4,5 \\
\hline Ensino Médio Completo & 79 & 35,4 \\
\hline Educação Superior Incompleta & 13 & 5,8 \\
\hline Educação Superior Completa & 22 & 9,9 \\
\hline Ignorado & 27 & 12,1 \\
\hline \multicolumn{3}{|l|}{ Profissão/Ocupação } \\
\hline $\begin{array}{l}\text { Forças Armadas, Policiais e Bombeiros } \\
\text { Militares }\end{array}$ & 1 & 0,4 \\
\hline $\begin{array}{l}\text { Membros do poder público, dirigentes de } \\
\text { empresas e gerentes }\end{array}$ & 4 & 1,7 \\
\hline Profissionais das ciências e das artes & 13 & 5,8 \\
\hline Técnico de nível médio & 12 & 5,3 \\
\hline Serviço de administração & 8 & 3,5 \\
\hline $\begin{array}{l}\text { Serviço de limpeza e vendedoras do } \\
\text { comércio }\end{array}$ & 76 & 34 \\
\hline Dona de Casa & 72 & 32,2 \\
\hline Ignorado & 37 & 16,5 \\
\hline
\end{tabular}

Em relação às características da gravidez atual, 168 (75,3\%) tiveram bebês a termo, $205(91,9 \%)$ realizaram o pré-natal, e 132 (59,2\%) realizaram mais de seis consultas. Quanto à paridade, 187 (83,8\%) eram multíparas, 215 (96,7\%) tiveram gestação única, e 119 (53,4\%) parto cesáreo. Com relação às complicações, 169 (75,8\%) mulheres as tiveram na gestação atual, com destaque para $48(28,4 \%)$ mulheres com diabetes mellitus gestacional (DMG) e 24 $(14,2 \%)$ com pré-eclâmpisa (Tabela 2).

Tabela 2 - Características da última gestação das mulheres de idade materna avançada que tiveram partos em um hospital escola do Sul do Brasil no ano de 2014. Curitiba/PR, Brasil, 2017. $(\mathrm{N}=223)$

\begin{tabular}{lcc}
\hline \multicolumn{1}{c}{ Variável } & $\mathrm{N}$ & $\%$ \\
\hline \multicolumn{1}{c}{ Idade Gestacional no Parto } & & \\
Pré-termo & 44 & 19,7 \\
A termo & 168 & 75,3 \\
Pós-termo & 11 & 5 \\
$\quad$ Consultas durante o pré-natal & & \\
Zero & 18 & 8,1
\end{tabular}




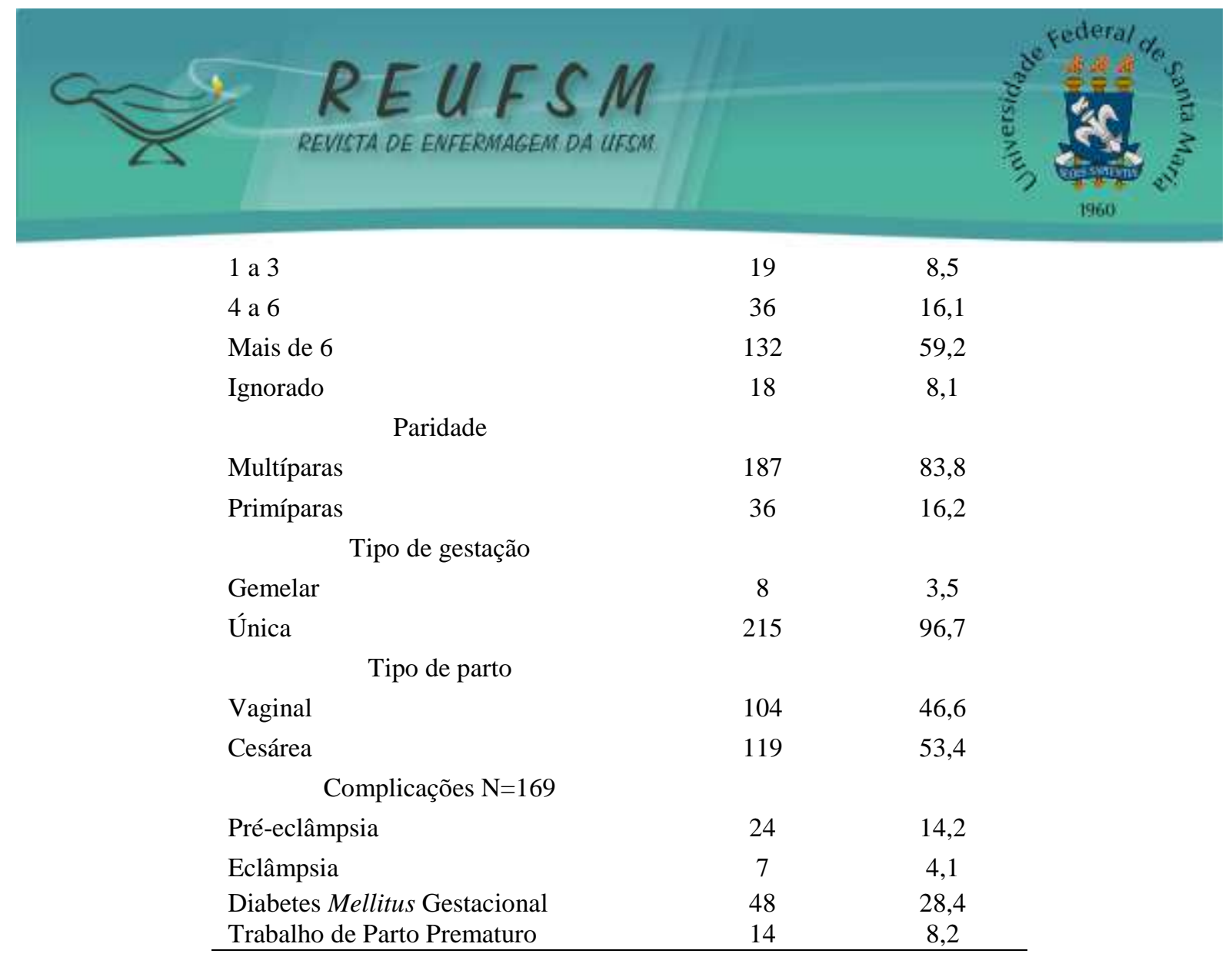

Entre as doenças pré-gestacionais, $20(19,4 \%)$ mulheres tinham hipotireoidismo, 8 (7,8\%) infecção pelo vírus da Imunodeficiência Humana (HIV) e 8 (7,8\%) apresentavam hipertensão arterial sistêmica. Das multíparas, 92 (49,1\%) não tiveram nenhuma complicação nas gestações anteriores. As que apresentaram, 21 (11,2\%) foram síndrome hipertensiva específica da gestação. A maioria das mulheres, ou seja, 192 (86,1\%), não teve bebês malformados (Tabela 3).

Tabela 3 - Características obstétricas anteriores à última gestação das mulheres de idade materna avançada que tiveram partos em um hospital escola do Sul do Brasil no ano de aldrigho2014. Curitiba/PR, Brasil, 2017. ( $=223)$

\begin{tabular}{lcc}
\multicolumn{1}{c}{ Variável } & $\mathrm{N}$ & $\%$ \\
\hline \multicolumn{1}{c}{ Doenças pré-gestacionais N=103 } & & \\
Hipotireoidismo & 20 & 19,4 \\
HIV: Vírus da Imunodeficiência Humana & 8 & 7,8 \\
Hipertensão Arterial Sistêmica & 8 & 7,8 \\
Depressão & 8 & 7,8 \\
Diabetes Mellitus Gestacional & 8 & 7,8 \\
Transtorno afetivo bipolar & 7 & 6,8 \\
Obesidade & 7 & 6,8 \\
$\quad$ Complicações nas gestações anteriores N=187 & & \\
Pré-Eclâmpsia & 14 & 7,5
\end{tabular}




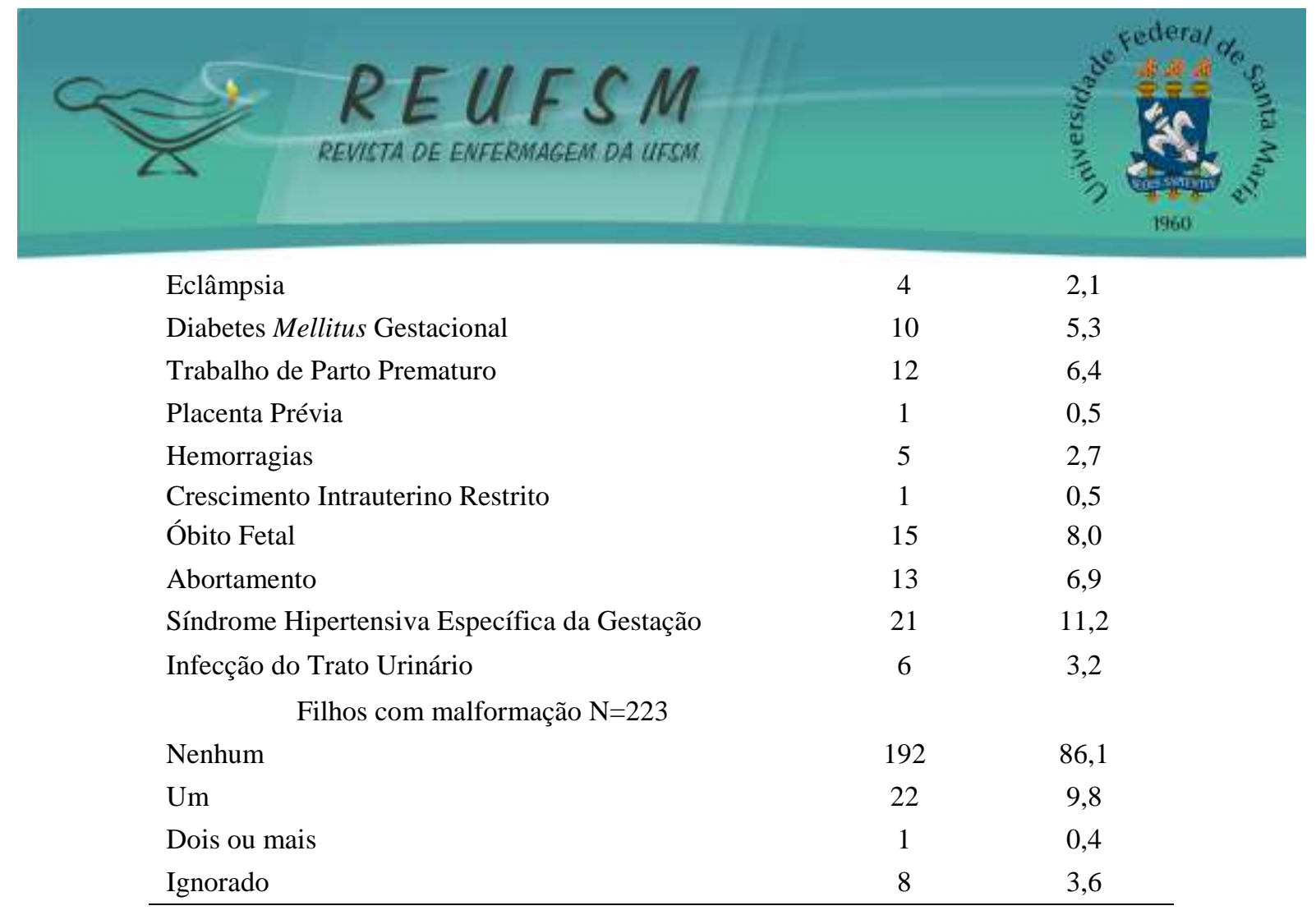

\section{DISCUSSÃO}

A gestação em idade avançada é uma realidade mundial, tanto nos países desenvolvidos como em desenvolvimento. Apresenta nuances atreladas a fatores biológicos, socioeconômicos e psicológicos importantes para a compreensão da mudança no perfil etário das gestações atuais. ${ }^{8}$

Nesse sentido, a literatura relata que o aumento do nível socioeconômico, o maior nível educacional e o aprimoramento frente ao mercado de trabalho têm se tornado prioridades na vida das mulheres, resultando na escolha e planejamento de gestações cada vez mais tardias. ${ }^{3,9-}$ ${ }^{10}$ Uma pesquisa mostra que nem sempre esse argumento é válido, pois 55\% das que tiveram a gestação em idade tardia não planejaram a gravidez. Entre aquelas que planejaram, apenas 2,5\% adiaram a gestação por motivos de estabilidade financeira e/ou no trabalho. ${ }^{11}$

Neste perfil sociodemográfico, a população pesquisada não tinha nível de escolaridade alto, tampouco uma profissão com boa remuneração para confirmar o argumento fornecido por muitos estudos de que as mulheres vêm adiando a gestação em busca de crescimento profissional e financeiro. ${ }^{5-6,9-10}$ Percebe-se que, no Brasil, esse perfil é diferenciado e pode estar relacionado com a falta de planejamento familiar, associada aos baixos níveis de escolaridade. Dessa forma, conhecer e reconhecer características sociodemográficas e econômicas que envolvem a gestante é importante para que o enfermeiro possa prestar um cuidado individualizado, considerando que a 


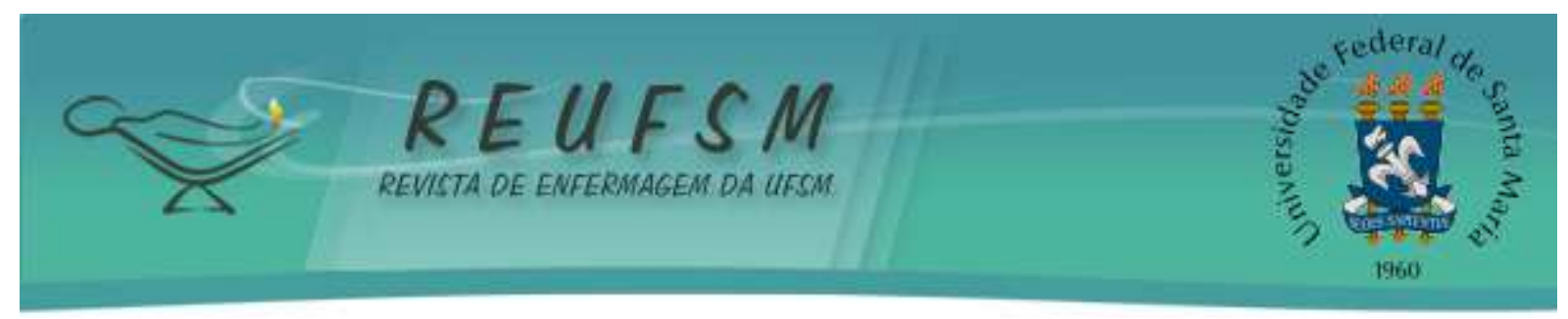

baixa escolaridade e a baixa renda são fatores que interferem na compreensão acerca da própria saúde o que pode refletir, por exemplo, na adesão ao pré-natal. ${ }^{12}$

Uma pesquisa retrospectiva realizada no Hospital Metropolitano de Sarandi/Paraná, entre 2007 e 2008, apresentou dados das gestantes com idade tardia similares aos encontrados no presente estudo quando relacionados ao estado civil e ao tipo de parto, em que a maioria das mulheres era casada e realizou parto cesárea. ${ }^{13}$ Ainda, a cor/raça predominante foi a branca confirmando dados do MS, os quais demonstram que 38,1\% das mulheres de cor branca têm filhos em idades mais avançadas. ${ }^{14}$

O município de residência das participantes mais frequente foi Curitiba. Apesar disso, foi possível perceber que muitas outras provinham diversas cidades do Paraná. Isso é explicado pelo fato de que o hospital é referência para gestante de alto risco e, frequentemente, recebe a população de cidades vizinhas ou, até mesmo, de outros Estados. Porém, a falta de infraestrutura em outras cidades pode gerar uma barreira para a mulher conseguir manter o acompanhamento necessário em uma gestação, como dificuldade para o agendamento da consulta e de transporte. ${ }^{15}$

Estudo retrospectivo realizado em Natal/Rio Grande do Norte, no ano de 2010, também se propôs a traçar o perfil sociodemográfico e gestacional e relacionar indicadores de bem-estar subjetivo com as variáveis idade, escolaridade e renda. Essa pesquisa apresentou dados muito semelhantes aos descritos nesta. As variáveis que corroboram com a presente foram a faixa etária dos 37 anos, a escolaridade, união consensual e a ocupação do lar. Os autores relatam que esses fatores, embora importantes, não influenciam significativamente na qualidade de vida das mulheres. $^{12}$

Em contrapartida, outro estudo que realizou uma revisão de literatura sobre qualidade de vida afirma que escolaridade e renda são alguns dos multifatores que estão diretamente ligados a esse conceito e que podem influenciar também no entendimento e nas questões de planejamento familiar. ${ }^{16}$

Nesse contexto, a enfermeira tem um importante papel no que concerne ao planejamento familiar, devendo suas atividades atingir todas as classes sociais e faixas etárias, sobretudo as mulheres com menor grau de escolaridade. Assim, desenvolver ações educativas de anticoncepção, planejamento familiar e compartilhamento de informações sobre a gestação em 


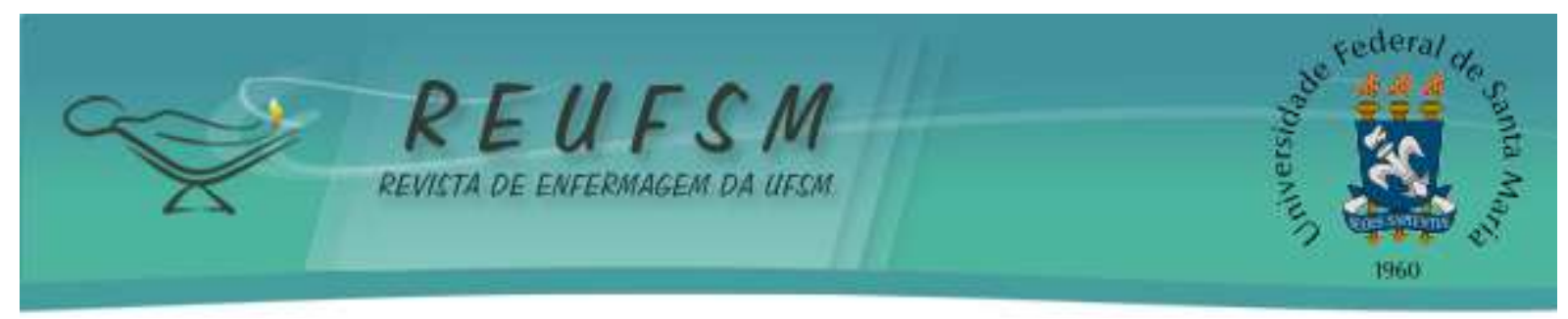

idade avançada com as mulheres dessa faixa etária pode informá-las sobre os riscos a que estão expostas, e dessa forma, o casal pode planejar conscientemente uma gestação em idade avançada.

No que diz respeito às profissões, destaca-se que as mulheres estão incluídas no grupo trabalhadoras do serviço de limpeza e vendedoras do comércio, de acordo com a Classificação Brasileira de Ocupações. As atividades que exigem graduação não foram frequentes, o que justifica o nível econômico baixo, como indicam outros estudos sobre o perfil das gestantes tardias. ${ }^{4,8}$

Predominaram, neste estudo, as gestantes multíparas, ao contrário de outro, o qual mostra que quase metade das gestantes de idade avançada eram primíparas. ${ }^{11}$ Em relação à idade gestacional no parto, 19,7\% tiveram recém-nascidos prematuros, diferindo de pesquisa realizada na Alemanha com 186 mulheres em idade avançada, que apresentou 28\% de partos pré-termo. ${ }^{17}$ Estudos apontam que a idade avançada é um fator de risco para nascimentos prematuros. ${ }^{17-18}$

Sobre a realização do pré-natal, $132(59,2 \%)$ puérperas realizaram mais de seis consultas, que configura o número mínimo preconizado pelo MS. ${ }^{2}$ Há evidências de que a procura pela assistência à saúde pode ser influenciada pela experiência gestacional anterior e que quando esta é negativa, a mulher tem a tendência de iniciar o pré-natal tardiamente, podendo apresentar menos consultas que o recomendado. ${ }^{15}$ Dessa forma, é importante que a enfermeira seja um agente de fortalecimento na vinculação das gestantes na atenção primária, com busca ativa por meio de visitas domiciliares frequentes. É preciso conscientizar os profissionais e a população para a prevenção de agravos referentes às gestações de risco, com a adesão precoce ao pré-natal, para que se chegue a resultados satisfatórios.

A gestação com feto único foi predominante em 215 (96,7\%), mas houve também gestações gemelares $(3,5 \%)$, apresentando dados similares a uma investigação que encontrou um índice de $92,5 \%$ de gestações únicas e 7,5\% de gestações gemelares. ${ }^{11}$ Em relação aos tipos de parto realizados, 119 (53,4\%) foram cesáreos e 104 (46,6\%), vaginais. O índice maior de partos cesáreos em gestantes com idade tardia também foi encontrado em outros estudos $^{1,6,13}$. Ainda, afirmam que a gestação em idade tardia acarreta o aumento do risco de parto cesárea, sendo este 1,23 vezes maior quando comparado a puérperas com menos de 35 anos. O risco dobra quando a pré-eclâmpsia está presente na gestação. ${ }^{13,19}$

É consenso na literatura que a idade materna avançada é fator de risco para o parto cesáreo, ${ }^{5-6,17}$ porém há também uma superestimação por essa via de parto devido ao medo do 


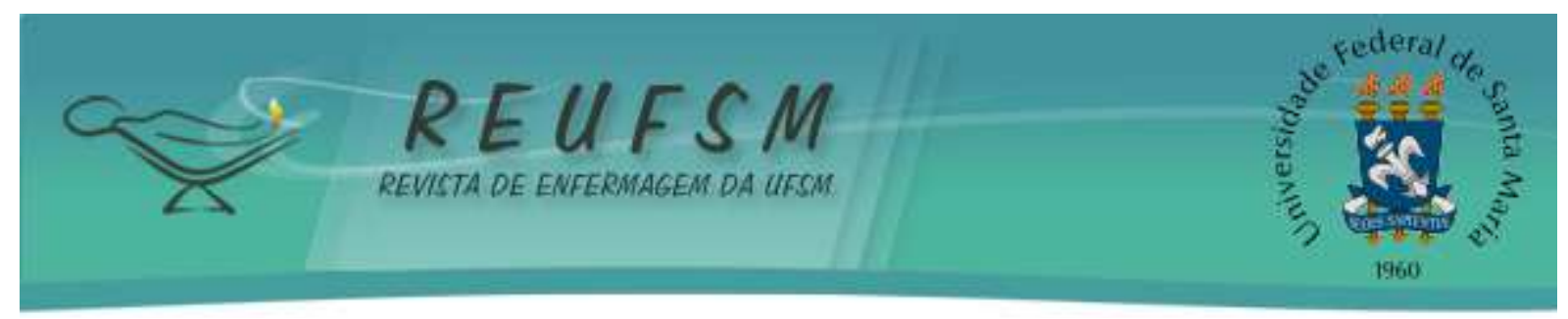

parto vaginal, seja por uma experiência negativa anterior ou por simplesmente não acreditar na naturalidade do corpo em parir. Não se deve excluir as indicações de cesárea amplamente discutidas, contudo, a idade não deve ser o único critério para a escolha dessa via. Nessa seara, durante o pré-natal, a enfermeira deve explorar esse campo de conhecimento, a fim de informar a mulher para que os partos cesáreos somente aconteçam quando necessários. ${ }^{20}$

No que concerne às complicações, observou-se que as mais encontradas no presente estudo foram DMG, pré-eclâmpsia e hipotireoidismo, assim como dados apontados em uma revisão de literatura. Nessa revisão, foi constatado que as primíparas de idade avançada apresentaram como complicações a eclâmpsia e a prematuridade. ${ }^{1}$

Outras complicações da gestação em idade tardia também são citadas na literatura, como macrossomia, trabalho de parto prematuro e óbito fetal. ${ }^{13}$ A macrossomia é uma complicação associada ao DMG e ao seu controle, por meio da aferição da glicemia, e o controle da dieta deve ser intensificado no segundo e terceiro trimestres gestacionais, pois esse período é o mais crítico para o surgimento da complicação. ${ }^{7}$ Dessa forma, a enfermeira deve prestar orientações quanto a esse distúrbio metabólico e intervir com o estímulo à alimentação balanceada, realização de atividades físicas frequentes e ingestão correta da medicação para auxiliar na manutenção da saúde da mãe e do bebê.

As doenças pré-gestacionais mais citadas foram hipotireoidismo, HIV, hipertensão arterial sistêmica e DMG. A somatização de doenças pré-gestacionais e complicações da gravidez atual podem agravar o quadro de saúde da gestante e colocar em risco o desenvolvimento físico e cognitivo do bebê. O HIV é uma de inúmeras infecções sexualmente transmissíveis que teve um aumento progressivo em gestantes. ${ }^{19} \mathrm{Na}$ presente pesquisa, nove mulheres viviam com o vírus HIV, e uma delas havia sido diagnosticada na atual gestação. No boletim do MS de 2015 já constava esse aumento, e uma das regiões do Brasil que obteve maior índice foi onde ocorreu esta investigação, com 30,8\% de gestantes notificadas no Sistema de Informação de Agravos de Notificação - SINAN de 2000 a 2015 na Região Sul. ${ }^{19}$

As complicações de saúde nas gestações anteriores mais presentes foram: doença hipertensiva específica da gestação; abortamento; óbito fetal; DMG; pré-eclâmpsia; e infecção do trato urinário. No grupo de gestantes tardias, a única doença em comum foi $\mathrm{DMG},{ }^{11}$ e em outro foram as infecções do trato urinário. ${ }^{4}$ 


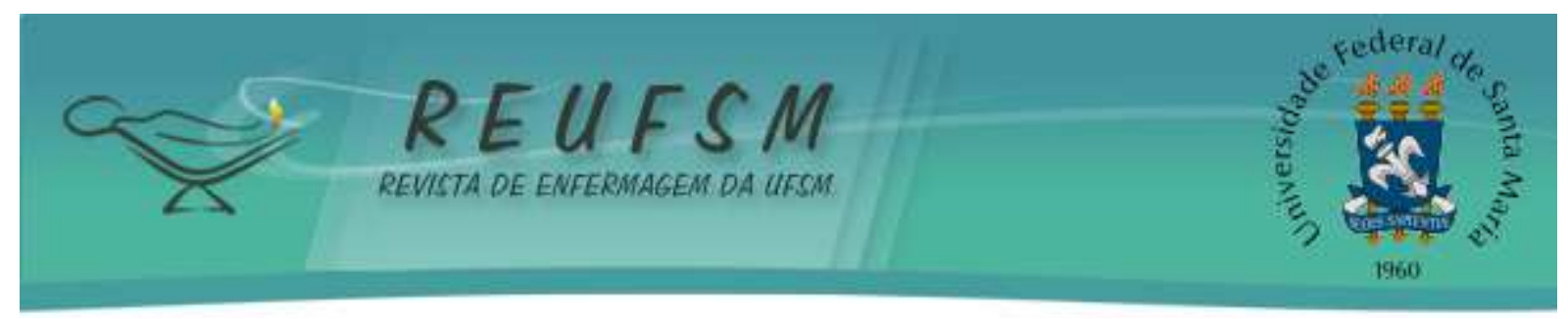

Em um estudo, pré-eclâmpsia, síndrome de HELLP e DMG apareceram como fatores de risco para o trabalho de parto prematuro em mulheres em idade avançada. ${ }^{17}$ Outro, que envolveu diversos países e 37.787 mulheres com idade igual ou maior a 35 anos, encontrou resultados adversos, incluindo morte materna e desfechos maternos graves em pacientes nessa faixa etária. ${ }^{18}$

Referente às malformações fetais, 23 mulheres $(10,2 \%)$ vivenciaram esta complicação. Uma pesquisa realizada em 2013, no Rio de Janeiro, com informações do banco de dados do ambulatório de pré-natal do Instituto Fernando Figueira da Fundação Osvaldo Cruz, aponta que o HIV e as malformações fetais foram associados às gestantes com menor rendimento financeiro e não a uma faixa etária específica. ${ }^{4} \mathrm{O}$ estudo que avaliou o perfil epidemiológico das malformações congênitas no Município de Cáceres/Mato Grosso, entre 2004 e 2009, encontrou um índice maior de malformações em bebês de gestantes com 21 a 30 anos, mas também estavam presentes em gestantes com 31 anos ou mais. O diagnóstico precoce da malformação torna-se essencial para que a equipe multidisciplinar de saúde tenha uma tomada de decisão quanto ao tratamento, quando esse é possível. ${ }^{21}$ Nesse sentido, o pré-natal tem uma importância fundamental, pois quando realizado de forma contínua e assídua, consegue-se obter um controle das comorbidades.

\section{CONSIDERAÇÕES FINAIS}

Dado o exposto, pode-se confirmar que a gestação em idade materna tardia apresentou uma alta porcentagem de complicações associadas à própria idade, mas também às doenças pré-gestacionais. Essas complicações devem ser controladas por meio do diagnóstico precoce durante o pré-natal de qualidade, com profissionais médicos e enfermeiros qualificados e comprometidos para atender essa população e suas especificidades.

O estudo revela que o perfil das gestantes tardias é de mulheres brancas, com baixa escolaridade e, consequentemente, ocupações laborais que não exigem nível avançado de estudo e baixa renda. Essas características vão de encontro ao que se encontra na literatura internacional acerca das gestações tardias. Já a literatura nacional confirma muitos achados da presente pesquisa, corroborando com a ideia de que as mulheres brasileiras que engravidam após os 35 anos, não correspondem ao perfil de países desenvolvidos.

Assim, é premente que a enfermagem preste um cuidado individualizado às gestantes tardias no intuito de identificar precocemente fatores de risco associados à idade e possibilite 


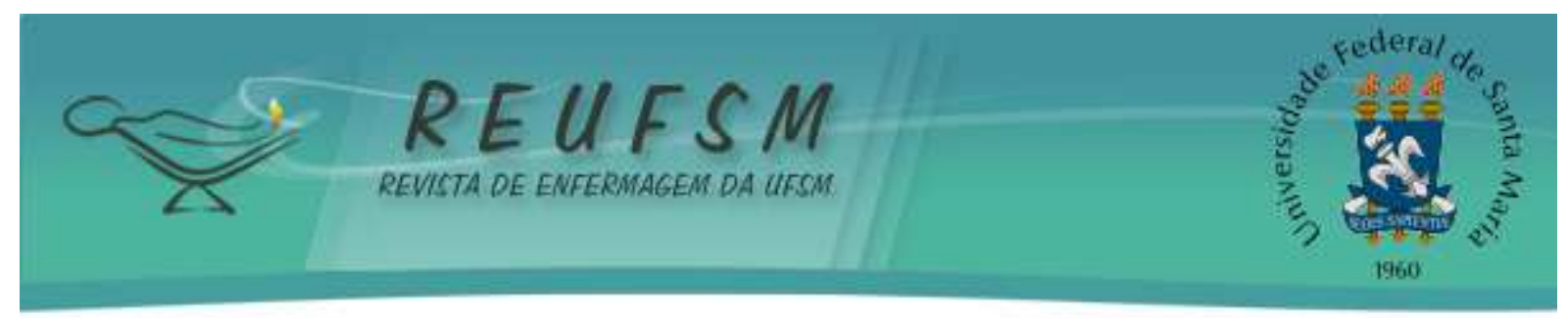

minimizar as consequências das possíveis complicações, pois ao conhecer essas características pode-se dar subsídios para ações de cuidado que sejam efetivas para essa população.

Entretanto, uma limitação deste estudo foi o não preenchimento correto e completo dos prontuários pelos profissionais. Destaca-se que muitos dados não estavam registrados no prontuário, por isso constaram como ignorados, principalmente em relação aos dados de prénatal e número de consultas.

Portanto, esta pesquisa se mostra relevante visto que trata de uma temática em crescimento em todo o mundo, a gestação em mulheres com idade maior de 35 anos. Sugerese que outras investigações sejam realizadas em diferentes cenários, a fim de buscar outras realidades para subsidiar as práticas dos profissionais da saúde, especialmente os enfermeiros, e proporcionar um cuidado individualizado e centrado nas necessidades de cada mulher.

\section{REFERÊNCIAS}

1. Gonçalves ZR, Monteiro DLM. Complicações maternas em gestantes com idade avançada. Femina [Internet]. 2012 [acesso em 2017 fev 16];40(5):275-79. Disponível em: http://files.bvs.br/upload/S/0100-7254/2012/v40n5/a3418.pdf.

2. Brasil. Ministério da Saúde. Secretaria de Atenção à Saúde. Departamento de Ações Programáticas Estratégicas. Gestação de alto risco: manual técnico. $5^{\mathrm{a}}$ ed. Brasília (DF): Ministério da Saúde; 2012.

3. Lopes MN, Dellazzana-Zanon LL, Boeckel MG. A multiplicidade de papéis da mulher contemporânea e a maternidade tardia. Temas Psicol [Internet]. 2014 [acesso em 2017 fev 16];22(4):917-28. Disponível em: http://pepsic.bvsalud.org/pdf/tp/v22n4/v22n04a18.pdf.

4. Xavier RB, Jannotti CB, Martins AC, Silva KS. Risco reprodutivo e renda familiar: análise do perfil de gestantes. Ciênc Saúde Colet [Internet]. 2013 [acesso em 2017 fev 16]; 18(4):1161-71. Disponível em: http://www.scielo.br/pdf/csc/v18n4/29.pdf.

5. Johnson J, Tough S. Delayed child-bearing. J Obstet Gynaecol Can [Internet]. 2012 [acesso em 2017 fev 16];34(1):80-93. Disponível em: https://sogc.org/wpcontent/uploads/2013/01/gui271CO1201E.pdf.

6. Fitzpatrick KE, Tuffnell D, Kurinczuk JJ, Knight M. Pregnancy at very advanced maternal age: a UK population-based cohort study. BJOG [Internet]. 2016 [acesso em $2017 \mathrm{fev} 16$ ]. Disponível em: http://onlinelibrary.wiley.com/doi/10.1111/1471-0528.14269/full.

7. Teixeira EC, Gurgel HM, Monteiro DLM. Gravidez em mulheres acima de 34 anos no Brasil - análise da frequência entre 2006 e 2012. Rev Hupe [Internet]. 2015 [acesso em 2018 jul 15];14(1):6-11. Disponível em: http://www.epublicacoes.uerj.br/index.php/revistahupe/article/view/16214/12993.

8. Aldrighi JD, Wall ML, Souza SRRK, Cancela FZV. The experiences of pregnant women at an advanced maternal age: an integrative review. Rev Esc Enferm USP [Internet]. 2016 


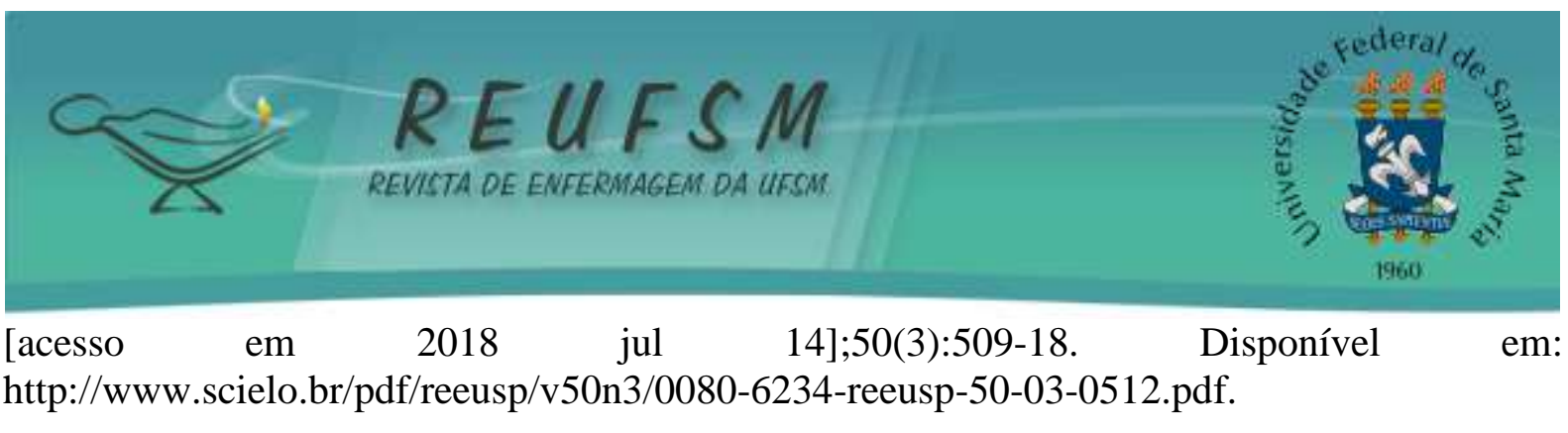

9. Budds K, Locke A, Burr V. "For some people it isn't a choice, it's just how it happens": Accounts of "delayed" motherhood among middle-class women in the UK. Fem Psychol [Internet]. 2016 [acesso em 2018 jun 04];26(2):170-87. Disponível em: http://fap.sagepub.com/content/26/2/170.full.pdf+html.

10. Welbi E, Chalmers A, Holly Y. Delayed motherhood: understanding the experiences of women older than age 33 who are having abortions but plan to become mothers later. Can Fam Physician [Internet]. 2012 [acesso em 2018 jul 15];58(10):e588-95. Disponível em: https://www.ncbi.nlm.nih.gov/pmc/articles/PMC3470537/pdf/058e588.pdf.

11. Ramos S, Faria N, Oliveira EA, Silva IS. O ajustamento materno na gravidez tardia: contributos para a prática de Enfermagem. Salutis Scientia [Internet]. 2012 [acesso em 2017 fev 16];4(2):24-39. Disponível em: http://www.salutisscientia.esscvp.eu/Site/Artigo.aspx?artigoid=30843.

12. Oliveira MAM, Sousa WPS, Pimentel JDO, Santos KSL, Azevedo GD, Maia EMC. Gestantes tardias de baixa renda: dados sociodemográficos, gestacionais e bem-estar subjetivo. Psicol Teor Prat [Internet]. 2014 [acesso em 2018 jul 15];16(3):69-82. Disponível em: http://pepsic.bvsalud.org/pdf/ptp/v16n3/06.pdf.

13. Gravena AAF, Sass A, Marcon SS, Pelloso SM. Outcomes in late-age pregnancies. Rev Esc Enferm USP [Internet]. 2012 [acesso em 2017 fev 16];46(1):15-21. Disponível em: http://www.scielo.br/pdf/reeusp/v46n1/en_v46n1a02.pdf.

14. Brasil. Ministério da Saúde. Secretaria de Vigilância em Saúde. Saúde Brasil 2014: uma análise da situação de saúde e das causas externas. Brasília (DF): Ministério da Saúde; 2015.

15. Viellas EF, Domingues RMSM, Dias MAB, Gama SGN, Filha MMT, Costa JV, et al. Prenatal care in Brazil. Cad Saúde Pública [Internet]. 2014 [acesso em $2017 \mathrm{fev} \mathrm{16];} \mathrm{30(Supl}$ 1):S85-S100. Disponível em: http://www.scielo.br/pdf/csp/v30s1/en_0102-311X-csp-30-s10085.pdf.

16. Pereira EF, Teixeira CS, Santos A. Qualidade de vida: abordagens, conceitos e avaliação. Rev Bras Educ Fís Esporte [Internet]. 2012 [acesso em 2017 fev 16];26(2):241-50. Disponível em: http://www.scielo.br/pdf/rbefe/v26n2/07.pdf.

17. Rendtorff R, Hinkson L, Kiver V, Dröge LA, Henrich W. Pregnancies in women aged 45 years and older - a 10-year retrospective analysis in Berlin. Geburtshilfe frauenheilkd [Internet]. 2017 [acesso em 2018 jun 04];77(3):268-75. Disponivel em: https://www.ncbi.nlm.nih.gov/pmc/articles/PMC5383433/.

18. Laopaiboon M, Lumbiganon $\mathrm{P}$, Intarut $\mathrm{N}$, Mori $\mathrm{R}$, Ganchimeg $\mathrm{T}$, Vogel JP, et al. Advanced maternal age and pregnancy outcomes: a multicountry assessment. RCOG [Internet]. 2014 [acesso em 2018 jun 04];121(Supl 1):49-56. Disponível em: https://obgyn.onlinelibrary.wiley.com/doi/epdf/10.1111/1471-0528.12659.

19. Brasil. Ministério da Saúde. Secretaria de Vigilância em Saúde. Departamento Nacional de DST e Aids. Boletim Epidemiológico Aids/DST; 2015. 


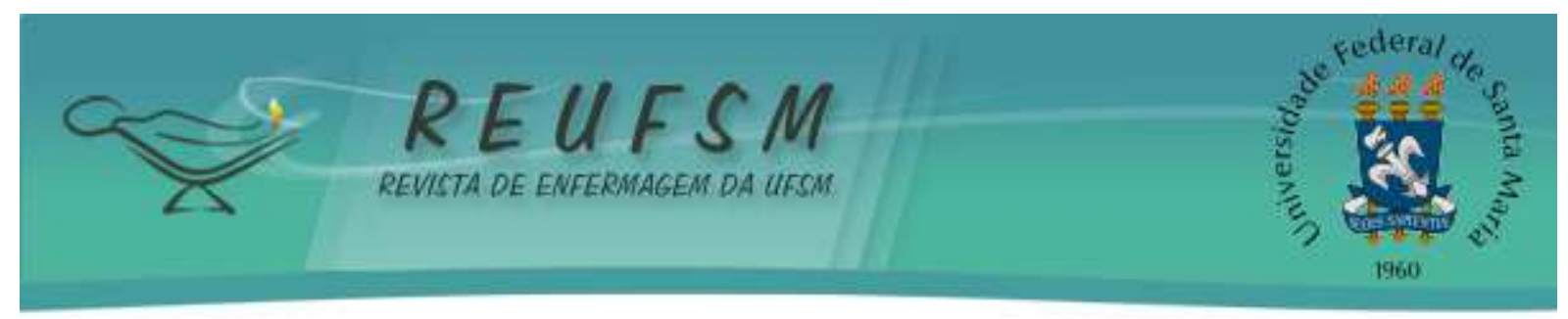

20. Aldrighi JD, Wall ML, Souza SRRK. Experience of pregnant women at an advanced age. Rev Gaúcha Enferm [Internet]. 2018 [acesso em 2018 jun 04];39:e2017-0112. Disponível em: http://seer.ufrgs.br/index.php/RevistaGauchadeEnfermagem/article/view/79573/46562.

21. Reis LLAS, Ferrari R. Congenital malformations: demographic profile of mothers and pregnancy conditions. Rev Enferm UFPE [Internet]. 2014 [acesso em 2017 fev 16];8(1):98106. Disponível em: https://periodicos.ufpe.br/revistas/revistaenfermagem/article/view/9611/9585.

Data de submissão: 20/02/2017

Data de aceite: $17 / 07 / 2018$

Autor correspondente: Juliane Dias Aldrighi

E-mail: juliane.aldrighi@gmail.com

Endereço: Avenida Lothário Meissner, 632 - Bloco didático II - Jardim Botânico

CEP: 80210-170 - Curitiba, PR, Brasil 